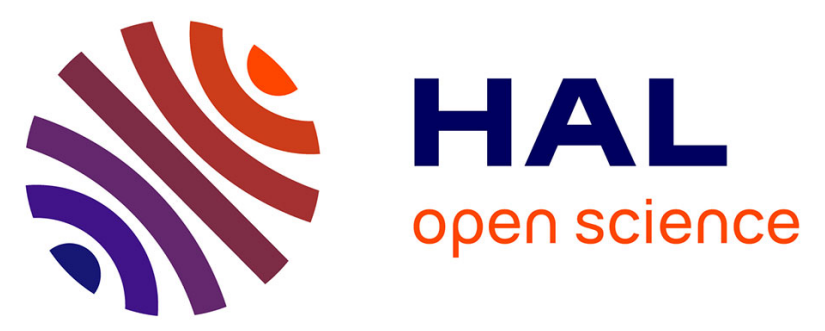

\title{
Comparative Case Study on the Self-Powered Synchronous Switching Harvesting Circuits With BJT or MOSFET Switches
}

Weiqun Liu, Adrien Badel, Fabien Formosa, Caiyou Zhao, Qiao Zhu, Guangdi $\mathrm{Hu}$

\section{To cite this version:}

Weiqun Liu, Adrien Badel, Fabien Formosa, Caiyou Zhao, Qiao Zhu, et al.. Comparative Case Study on the Self-Powered Synchronous Switching Harvesting Circuits With BJT or MOSFET Switches. IEEE Transactions on Power Electronics, 2018, 33 (11), pp.9506 - 9519. 10.1109/tpel.2018.2790965 . hal-01901537

\section{HAL Id: hal-01901537 \\ https://hal.science/hal-01901537}

Submitted on 23 Oct 2018

HAL is a multi-disciplinary open access archive for the deposit and dissemination of scientific research documents, whether they are published or not. The documents may come from teaching and research institutions in France or abroad, or from public or private research centers.
L'archive ouverte pluridisciplinaire $\mathbf{H A L}$, est destinée au dépôt et à la diffusion de documents scientifiques de niveau recherche, publiés ou non, émanant des établissements d'enseignement et de recherche français ou étrangers, des laboratoires publics ou privés. 


\title{
Comparative case study on the self-powered synchronous switching harvesting circuits with BJT or MOSFET switches
}

\author{
Weiqun $\mathrm{Liu}^{1}$, Adrien $\mathrm{Badel}^{2}$, Fabien Formosa ${ }^{2}$, Caiyou Zhao ${ }^{3}$, Qiao Zhu ${ }^{1}$ and Guangdi $\mathrm{Hu}^{1}$ \\ 1 School of Mechanical Engineering, Southwest Jiaotong University, Chengdu, China 610031 \\ 2 Laboratory SYMME, Université Savoie Mont Blanc, Annecy le Vieux, France 74944 \\ 3 School of Civil Engineering, Southwest Jiaotong University, Chengdu, China 610031
}

\begin{abstract}
Self-powered realization for synchronous switching circuits is a hot spot for piezoelectric vibration energy harvesting. Two typical kinds of switches, bi-polar junction transistor (BJT) or metal oxide semiconductor field effect transistor (MOSFET) are popularly used without a clear understanding about the performance difference. In this paper, comparative investigations about adopting these two types of switches based on a case study are performed. It is analyzed that the performance difference comes from three aspects: the gate parasitic capacitance, the turning-on threshold and the driven mechanism. In particular, the third one imposes a critical influence on the performance. Investigations are performed on the self-powered synchronous switching circuit of two possible methods: with electronic breakers or with external control units. The results from simulations and experiments show that the current-driven mechanism limits the available performance in the case of the BJT switch in comparison with the case of the MOSFET switch. The difference is especially obvious for the cases of large piezoelectric capacitance and open-circuit voltage. A preliminary design guideline is concluded that the MOSFET is probably a better choice with the same voltage and current ratings in most cases.
\end{abstract}

Keywords: Piezoelectric generator; Synchronous switching circuit; BJT; MOSFET; Current-driven; Voltage-driven

\section{Introduction}

As one of the promising techniques for sustainable power source, piezoelectric generator can scavenge energy from ambient vibrations [1]. The researches about piezoelectric energy harvesting generally focus on two subjects: (1) the improvement on the mechanical structure to increase power density [2] or operation bandwidth [3-5] and (2) the study about the electronic interface 
circuits of high performance to get the most power for the generator.

Generally, the interface circuit is used for regulating the alternative current (AC) from the generator to direct current (DC) and delivering the extracted power to the load. For this purpose, the standard circuit simply composed of a rectifier and a capacitor was firstly proposed [6]. However, a great part of energy is not extracted but returned to the dynamic system due to the limitation of the rectifier. This energy return phenomenon [7] makes its performance not good in low electromechanical coupling cases [8]. To increase the harvested power, many nonlinear synchronous switching harvesting techniques were developed, for instance, synchronized switch harvesting on inductor (SSHI) [9-11] and synchronous electric charge extraction (SECE) [12]. These circuits can significantly increase the harvested power in comparison with the standard circuit, especially in the case of low electromechanical coupling level. Moreover, as another desired property for the interface circuit, low load dependence is also observed. Further improvements were proposed on SSHI such as double synchronized switch harvesting (DSSH) [13], SSHI with magnetic rectifier (SSHI-MR) [14], enhanced SSHI [15] etc. or on SECE such as optimized SECE (OSECE) [16], tunable SECE [17] etc. Different features, such as better power or bandwidth etc. are provided with these circuits.

For these synchronous switching circuits, a critical issue in the way is the self-powered realization for the switching control. A variety of approaches have been studied, including mechanical switches [18-21], velocity control [22], integrated circuits [23-25] or electronic breakers [26-28]. Among them, the mechanical switch relies on the structure's dynamic motion to connect and disconnect, and it has the advantage of simplicity and fewer electronic components requirements. However, it is sometimes not so reliable because the switching position is not so adaptive and accurate. The velocity control utilizes an additional piezoelectric element as the sensing unit and drives the switches with an analog compartor, implying special fabrication requirements on the generator and slightly complex circuit [22]. The integrated circuit is the smartest with the possible realization of advanced control strategy but the most complex. The switch is controlled by either a clock divider [23] or a microcontroller [25] etc. Generally, the velocity control and the integrated circuit can be viewed together as the self-powered switching circuits with external control units. For this case, the switching instant can be precisely controlled with negligible phase lag. Compared with the former methods, the electronic breaker composed of an envelope and a comparator receives great interests due to its simplicity and reliability. It was firstly proposed for SSHI [26] and subsequently extended for other synchronous switching circuits [28]. In the self-powered 
switching circuits, the electronic breakers powered by the piezoelectric source automatically detects the piezoelectric voltage maxima or minima and takes action to turn the switch on or off properly. The harvested power is strongly related to the available open-circuit voltage magnitude and the phase lag between the switching actions and the corresponding piezoelectric extrema in the self-powered circuits $[27,31]$. In order to find out the relationship between the self-powered circuit's performance and the used components, an early analysis was conducted by Lallart and Guyomar including the voltage drop on the diode and the transistor [26]. Nevertheless, other components in the circuit were not accounted and the phase lag was assumed to be constant. Liang and Liao proposed a model with an improved analysis which contains the influence of the envelope capacitor and the leakage resistance of the piezoelectric element [27]. The phase lag induced by the diode and the transistor in the electronic breaker is analyzed. However, the RC delay introduced by the envelope detector's resistor and capacitor is neglected. The phase lag effect induced by charging the switch's parasitic capacitance with the envelope capacitance to turn on the switch is not studied either. More recently, a comprehensive modeling and analysis has been performed to take these two factors in consideration with more accurate results obtained [29].

Easy to find, as one of the most important components of the self-powered circuit, the switch plays a critical role in the circuit performance in the self-powered switching circuits with electronics. Even for the velocity control method and the integrated circuit approach in which the switching operations are controlled by external control units with phase lag negligible, the switches still affects circuit efficiency. However, few investigations have been performed to detail the switch's influence and the design guideline of the switch type selection from two typical available options: bi-polar junction transistor (BJT) or metal oxide semiconductor field effect transistor (MOSFET). Both of them have been popularly used, for instance, BJT in [26-27] and MOSFET in $[22-25,28]$. It is interesting to investigate when BJT or MOSFET is better.

Due to the distinguished working principle, there are mainly three different aspects between BJT and MOSFET to be considered when used as switches in the self-powered synchronous switching harvesting circuits: the parasitic capacitance, the turning-on threshold and the driven mechanism. The former two will significantly affect the phase lag in the approach of electronic breakers. Meanwhile, the current-driven BJT and the voltage-driven MOSFET imposes variations in the circuit's energy transfer efficiency with electronic switches. They will together affect the selection of other circuit components and performance 
discrepancy is expected to use the two type switches respectively. Consequently, comparison investigations about adopting these two types of switches are necessary and useful for the circuit design. For this purpose, a case study has been performed in this paper using the BJT TIP31C and the MOSFET Si2392ads which have comparable voltage and current ratings.

This paper is organized as follow: section 2 presents the introduction on the principle and operation of the self-powered OSECE (SP-OSECE) circuit with the electronic breaker. The SP-OSECE circuit is selected here for investigations as a representative of the synchronous switching circuits due to the easy feasibility and low load dependence [16]. It facilitates the discussion by choosing a constant load without affecting the generality. Section 3 presents the comparative investigations about the two switches types in the SP-OSECE circuit with electronic breakers and section 4 further performs the comparison of the two switches in the SP-OSECE circuit with other switching control approaches instead of electronic breakers. In the end, some conclusions are given in section 5.

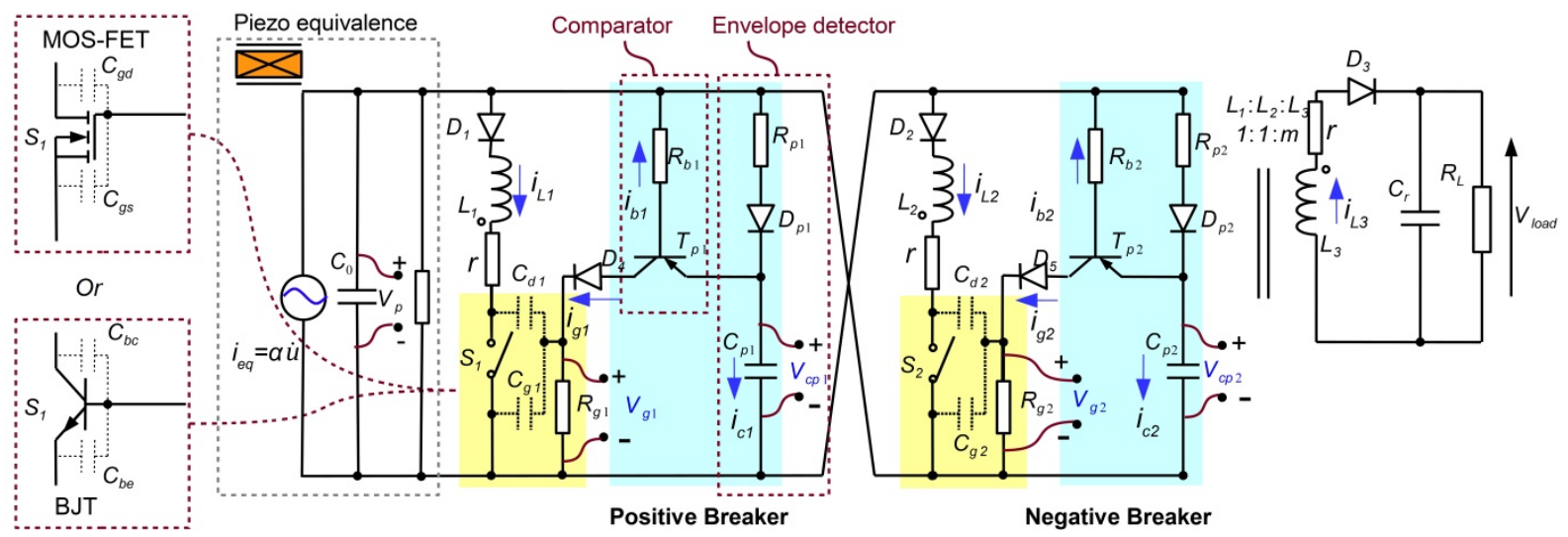

Fig. 1. SP-OSECE circuit with two possible switch types of MOSFET and BJT.

\section{Principle of the SP-OSECE circuit with electronic breakers}

Considering that the analysis about the SP-OSECE circuits is fully described in [28, 29], the principle will be briefly reminded for the readability. Fig. 1 presents the SP-OSECE circuit which mainly consists of two identical electronic breakers, two switches and a fly-back transformer composed of three windings $L_{1}, L_{2}$ and $L_{3}$ with a 1:1:m turn ratio. The electronic breaker including an envelope detector and a comparator is used to produce the desired switching control signal at the piezoelectric voltage extreme position while the fly-back transformer is deployed for transferring the energy to the load. Regarding the switches $S_{1}$ and $S_{2}$, they can be implemented with MOSFETs or BJTs with other components unvaried. Two capacitors $C_{g i}(i=1,2)$ and $C_{d i}$ for each switch are introduced to represent the parasitic capacitance of the MOSFET $\left(C_{g s}, C_{g d}\right)$ and the BJT( $\left.C_{b e}, C_{b c}\right)$ for the 
consideration that $C_{g s}$ or $C_{b e}$ plays an important role in the circuit performance [29]. $C_{g d}$ or $C_{b c}$ is also plotted for the completeness despite the insignificant effect on the circuit. Besides, two gate resistors $R_{g i}$ are used at the control end of the switches. The usage of $R_{g 1}\left(R_{g 2}\right)$ is based on two reasons: (1) discharging the reversely accumulated charge on $C_{g 1}\left(C_{g 2}\right)$ last half period and (2) discharging $C_{g 1}\left(C_{g 2}\right)$ to zero to turn off the switch $S_{1}\left(S_{2}\right)$ before the operation of $S_{2}\left(S_{1}\right)$. In this way, the switching operation is facilitated without the needs for additional neutralization charge from $C_{p i}$. Notably, a frictional resistor $r$ is introduced in each inductor branch to stand for the parasitic serial resistance loss on the transformer and the diodes.
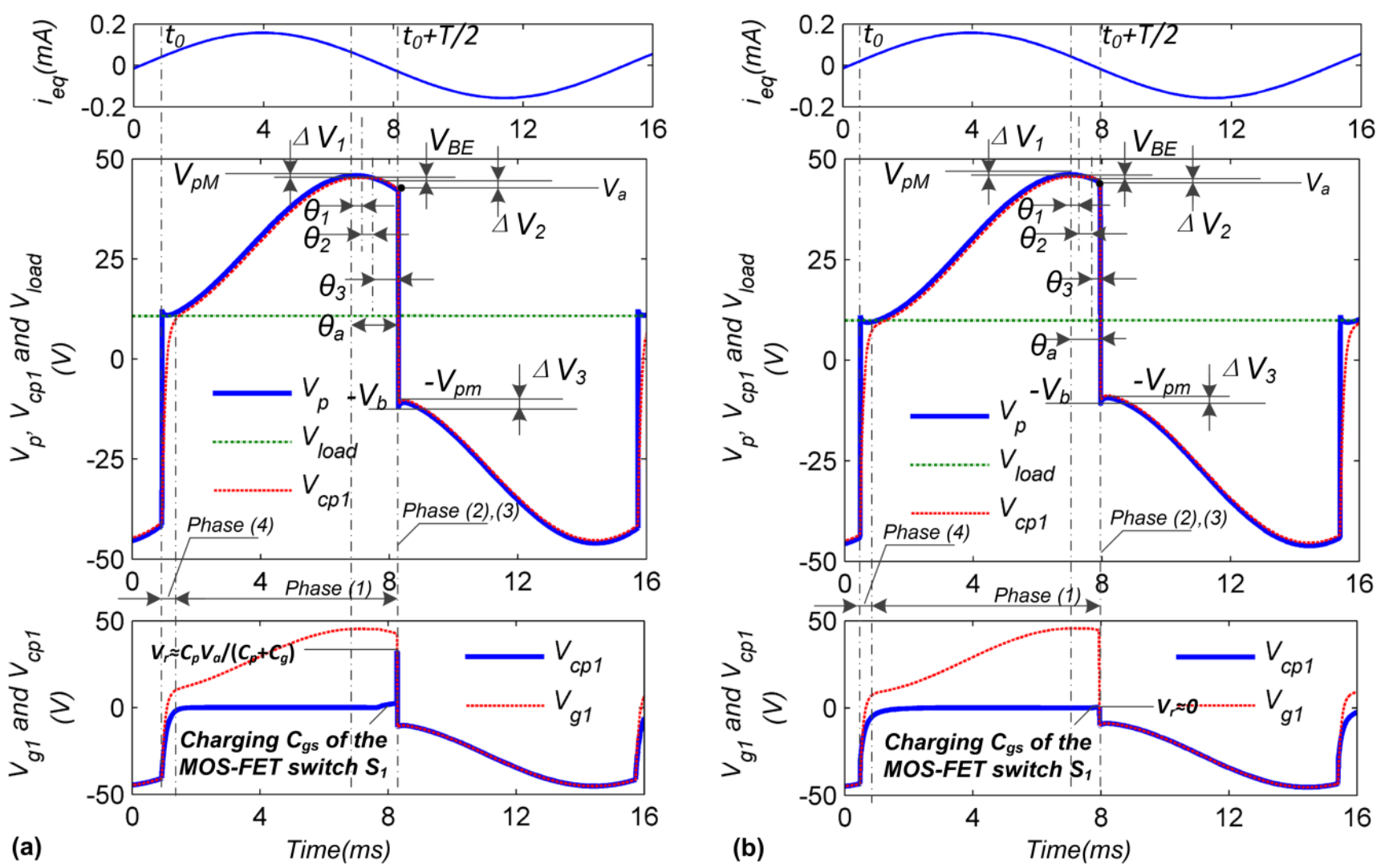

Fig. 2. Simulated voltage waveforms of the SP-OSECE circuit with two different switches and other components identical: (a) MOSFET Si2392; (b) BJT TIP31C.
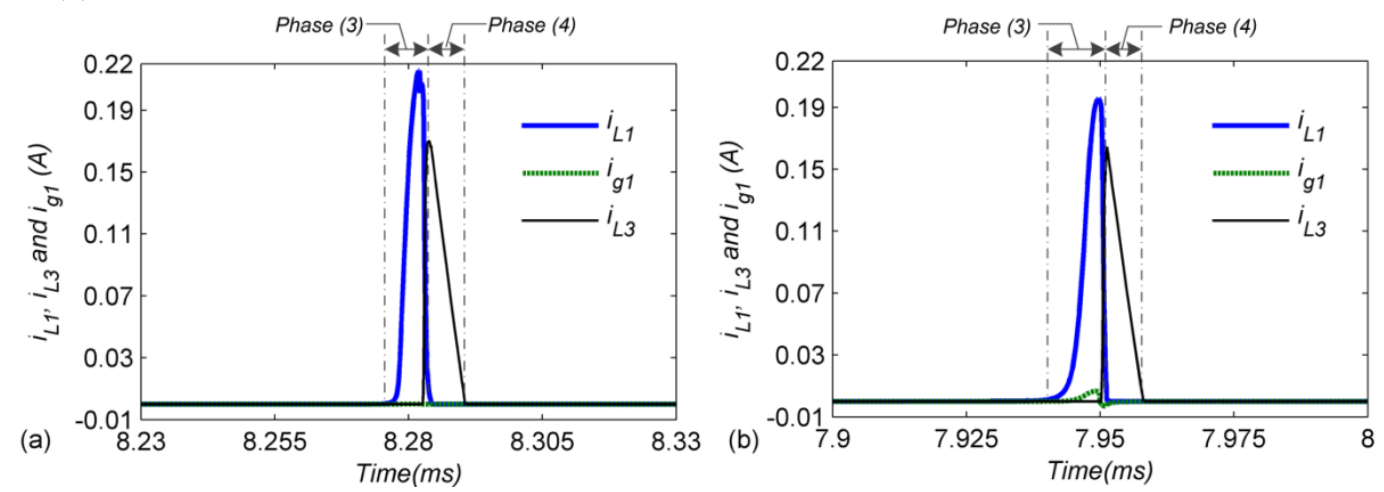

Fig. 3. Simulated current waveforms of the SP-OSECE circuit: (a) MOSFET Si2392; (b) BJT TIP31C.

In the circuit, the piezoelectric element is equivalent to a current source $i_{e q}$ in parallel with an intrinsic capacitance $C_{0}$ and a leakage resistor $R_{0}$. Considering that this paper is devoted to the circuit investigation, the piezoelectric generator is assumed to be 
subjected to a sinusoidal motion of constant displacement amplitude $u_{M}$ and $u=u_{M} \sin \omega t$ and we have

$$
i_{e q}=\alpha \dot{u}=\alpha \omega u_{M} \cos \omega t
$$

according to the piezoelectric constitutive equation. Here, $\alpha$ is the force-factor of the piezoelectric generator, $\omega$ is the vibration frequency and $u$ is the displacement. Then the analysis about the piezoelectric generator with the SP-OSECE circuit can be performed completely from the electric aspect. Therefore, the current source $i_{e q}$ is determined and a numerical model can be constructed in the LT-SpiceC software. For comparison purpose, the waveforms of the SP-OSECE circuit in Fig. 2 and Fig. 3 are obtained with LT-Spice $\odot$ software using the MOSFET Si2392ads and the BJT TIP31C as switches for $R_{p 1}=R_{p 2}=R_{p}=200 \mathrm{k} \Omega$ and $C_{p 1}=C_{p 2}=C_{p}=0.5 \mathrm{nF}$ with other components listed in Table 3.

As indicated in Fig. 2 and Fig. 3, the operation of the SP-OSECE circuit can be divided into four phases for each half-period between two switching operations: (1) natural charging; (2) voltage inversion; (3) energy transfer; (4) charge neutralization. Considering the positive half-period $t_{0} \rightarrow t_{0}+T / 2$ ( $T=2 \pi / \omega$ is the period) as shown in Fig. $2, S_{1}$ and $S_{2}$ are opened at the beginning of the natural charging phase. Both $\mathrm{LC}$ branches are blocked and the current $i_{e q}$ charges $C_{0}$ with $V_{p}$ increasing. At the meantime, the detector capacitor $C_{p 1}$ is also charged through $R_{p 1}$ and $D_{1}$ while $C_{p 2}$ and $C_{g 2}$ are charged through $T_{p 2}$ and the base resistor $R_{b 2}$. As $V_{p}$ reaches the extreme $V_{p M}, V_{c p 1}$ reaches the peak value $V_{p M}-\Delta V_{1}$ subsequently with some delay due to the RC circuit composed of $R_{p 1}$ and $C_{p 1}$. The peak is then preserved by the envelope detector and $V_{p}$ starts to decline. When $V_{p}$ goes smaller than $V_{p M^{-}}-\Delta V_{1}-V_{B E}$ ( $V_{B E}$ is the base-emitter voltage of $T_{p 1}$ and $\Delta V_{1}$ is the voltage drop on $R_{p 1}$ and $D_{1}$ ), the comparator $T_{p 1}$ turns on and the current from $C_{p 1}$ charges $C_{g 1}$ to make $V_{g 1}$ greater than the threshold voltage $V_{t h}\left(V_{g s-t h}\right.$ for MOSFET and $V_{B E}$ for BJT). During this process of turning on the switch, $V_{p}$ further decreases to $V_{p M}-\Delta V_{1}-V_{B E}-\Delta V_{2}$ and the nature charging phase (1) ends. Then the voltage inversion phase (2) starts in the form of LC oscillation $\left(L_{l}\right.$ and $\left.C_{0}\right)$. Due to the configuration of the fly-back transformer, the energy stored in the LC circuit is not transferred at the first quarter of the LC oscillation due to the reversed bias of $D_{3}$ because the induced voltage on $L_{3}$ is negative. As the LC oscillation continues and $V_{p}$ is reversely charged to $-\left(V_{\text {load }}+V_{D}\right) / m\left(V_{D}\right.$ is the voltage drop on the diode), the voltage on $L_{3}$ at the secondary side becomes larger than $V_{\text {load }}+V_{D}$. The SP-OSECE circuit enters phase (3) with the energy stored in the transformer transferred to the load. Afterwards, the charge neutralization phase (4) begins due to the voltage difference between $C_{0}, C_{p 1}, C_{p 2}$ and $C_{g 1}$. The current from $C_{0}$ starts to reversely charge $C_{p 1}, C_{g 1}$ through $\left(T_{p 1}, R_{b 1}\right)$, and $C_{p 2}$ through 
$\left(R_{p 2}, D_{2}\right)$. As a result, $V_{g 1}$ becomes negative and $S_{1}$ is closed. It is represented by RC process as indicated in Fig. 2 corresponding

to the time constant $\tau_{1}$ and $\tau_{2}$ respectively. As a result, a voltage retreat towards zero for $V_{\mathrm{p}}$ due to the charge neutralization process can be observed in Fig. 2. At the meantime, the accumulated charge on $C_{g 2}$ with the positive $V_{g 2}\left(S_{2}\right.$ is closed when $V_{g 2}$ is negative and lower than the threshold) begins to discharge through $R_{g 2}$. It facilitates the switching on of $S_{2}$ since the charge from $C_{p 2}$ is only required to charge $C_{g 2}$ to $-V_{g s-t h}$ from zero instead of a relatively large positive value. With the positive half-period ending, the SP-OSECE circuit starts to operate similarly for the subsequent negative half-period.

\section{Comparative investigations on the SP-OSECE circuit with electronic breakers}

\subsection{Analysis}

In spite of the similar operations of the SP-OSECE circuit with the two different switches, it is noticed that the circuit performance represented by the load voltage $V_{\text {load }}$ with the same $R_{L}$ in Fig. 2 is different, 10.8V for the MOSFET and 9.9V for the BJT. The maximum piezoelectric voltage $V_{p M}$ and the switching voltage $V_{g l}$ in two cases differs as well. It implies that the switch type plays an important role on the circuit performance, thus it is meaningful to perform studies about the switch's influence for the improvement of the circuit design.

Using the model derived in [29], the SP-OSECE power performance can be written as

$$
P_{S P_{-} O S E C E}=\frac{V_{\text {load }}^{2}}{R_{L}} \approx \frac{\eta f C_{0} m^{2} V_{a}^{2}}{\left(\eta f C_{0} R_{L}+m^{2}\right)}
$$

in which $f=\omega / 2 \pi, \eta$ is the energy transfer efficiency of the circuit related to the circuit quality relies on the used components (diodes, switches, transformer, transistors etc.) and the working conditions such as the excitation level and the load situation etc. [30], $m$ is the turn ratio and $V_{a}$ is the piezoelectric voltage at the start of the voltage inversion phase:

$$
V_{a} \approx V_{p m}+2 V_{o c} \cos \theta_{a} \approx \frac{V_{\text {load }}}{m}-\Delta V_{3}+2 V_{o c} \cos \theta_{a}
$$

where $\theta_{a}$ is the phase lag between the switching instant and the peak position $V_{p M}, \Delta V_{3}$ is the voltage retreat variation due to the charge neutralization as indicated in Fig. 2 and $V_{o c}$ is the generator's open-circuit voltage magnitude. Using eq. (2), we have

$$
V_{a}=\frac{-\Delta V_{3}+2 V_{o c} \cos \theta_{a}}{1-\sqrt{\frac{\eta f C_{0} R_{L}}{\eta f C_{0} R_{L}+m^{2}}}}
$$

and the open-circuit voltage magnitude can be expressed as 


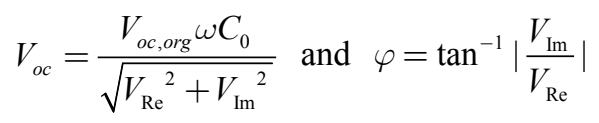

with

$$
\begin{aligned}
& V_{o c, \text { org }}=\frac{\alpha u_{M}}{C_{0}} \\
& V_{\mathrm{Re}}=\omega C_{0}+\frac{\omega\left(C_{g}+C_{p}\right)}{1+\omega^{2}\left(C_{g}+C_{p}\right)^{2} R_{b}^{2}}+\frac{\omega C_{p}}{\omega^{2} C_{p}^{2} R_{p}^{2}+1} \\
& V_{\operatorname{Im}}=\frac{1}{R_{0}}+\frac{\omega^{2}\left(C_{g}+C_{p}\right)^{2} R_{b}}{1+\omega^{2}\left(C_{g}+C_{p}\right)^{2} R_{b}^{2}}+\frac{\omega^{2} C_{p}^{2} R_{p}}{\omega^{2} C_{p}^{2} R_{p}^{2}+1}
\end{aligned}
$$

Here, $V_{o c, \text { org }}$ represents the generator's original open-circuit voltage with $R_{0}$ neglected and the SP-OSECE circuit disconnected, $R_{b 1}=R_{b 2}=R_{b}$ is the base resistance of the comparator transistor $T_{p i}$ and $\varphi$ is the phase difference between $V_{p}=V_{p M}$ and $i_{e q}=0$. Eq. (4) is obtained on the basement that the SP-OSECE can be simplified as a RC network composed of $R_{p 1}, C_{p 1}, C_{g s 1}, C_{g s 2}, C_{p 2}$ and $R_{b 2}$ in the natural charging phase. This can be deduced from the circuit's operation principle in Fig. 1 . When $\omega R_{p} C_{p}<<1$ and $\omega R_{b}\left(C_{p}+C_{g}\right)<<1$ are satisfied for the usual case, eq. (5) can be further simplified as

$$
V_{o c} \approx \frac{C_{0} V_{o c, o r g}}{C_{0}+2 C_{p}+C_{g}}
$$

As shown in Fig. 2, $\theta_{a}$ can be further divided into three parts: $\theta_{1}$ from the envelope detector's RC circuit delay $\left(R_{p i}\right.$ and $\left.C_{p i}\right)$, $\theta_{2}$ from the voltage drop to make the comparator turn on, and $\theta_{3}$ from the charging process to turn on the switch. It holds

$$
\begin{aligned}
& \theta_{1}+\theta_{2}=\cos ^{-1}\left(1-\frac{\Delta V_{1}+V_{B E}}{V_{o c}}\right)=\cos ^{-1}\left(\frac{1-V_{D} / V_{o c}}{\sqrt{1+\omega^{2} R_{p} C_{p}}}-\frac{V_{B E}}{V_{o c}}\right) \\
& \theta_{3}=\theta_{a}-\left(\theta_{1}+\theta_{2}\right)=\cos ^{-1}\left(\frac{1-V_{D} / V_{o c}}{\sqrt{1+\omega^{2} R_{p} C_{p}}}-\frac{V_{B E}}{V_{o c}}-\frac{\Delta V_{2}}{V_{o c}}\right)-\cos ^{-1}\left(\frac{1-V_{D} / V_{o c}}{\sqrt{1+\omega^{2} R_{p} C_{p}}}-\frac{V_{B E}}{V_{o c}}\right)
\end{aligned}
$$

in which $\Delta V_{1}$ is the voltage drop on the diode $D_{p i}$ and the envelope detector resistor $R_{p i}$, and

$$
\Delta V_{2} \approx C_{g} V_{t h} / C_{p}+V_{t h} \theta_{3} /\left(\omega R_{g}\right)
$$

is the voltage drop of $V_{p}$ during the charging of $C_{g i}$ until the corresponding switch is turned on. Obviously, $\theta_{1}$ and $\theta_{2}$ are almost not affected by $C_{g}$ and $V_{t h}$ except the slight and indirect influence by $V_{o c}$. However, $\theta_{3}$ is directly related to $C_{g}$ and $V_{t h}$ as seen in eq. (7) and eq. (8) since it determines the time to charge $C_{g}$ over $V_{t h}$ and the voltage drop $\Delta V_{2}$. In particular, the last term at the right side of eq. (8) accounts for the leakage current through the gate resistor $R_{g i}=R_{g}$ for this duration, implying that $V_{t h}$ imposes more influence than $C_{g}$.

With the same components except the switches, the values of $m, R_{p}, R_{b}, C_{0}, R_{L}, f$ and $C_{p}$ are identical for both cases of 
MOSFET and BJT switches. By carefully observing eq. (2) and (4), it can be deduced that the performance difference is only related to $V_{o c}, \Delta V_{3}, \theta_{a}$ and $\eta$. Analysis is thus performed on these variables for the two cases of BJT and MOSFET in order to understand the influence of the switch type. Before conducting the comparison, three pairs of BJT and MOSFET switches which have similar max allowed $V_{D S}$ (or $\left.V_{C E}\right) / I_{D}$ (or $I_{C}$ ) are chosen to obtain a preliminary knowledge about the difference of the two switch types and the parameters are listed in Table 1 . The ratings vary from $100 \mathrm{~V} / 3 \mathrm{~A}, 60 \mathrm{~V} / 2 \mathrm{~A}$ to $20 \mathrm{~V} / 1 \mathrm{~A}$ in the around. Considering the power electronics applications, the switches with the current rating below 1A are not considered here. However, due to the difficulty to find the BJT and MOSFET switch with the exactly same rating, the MOSFET switch Si2392ads has a current rating of $3.1 \mathrm{~A}$ for $100 \mathrm{~V}$ grade and Si2308bds has a current rating of $1.9 \mathrm{~A}$ for the $60 \mathrm{~V}$ grade with the ambient temperature $\mathrm{TA}=25^{\circ} \mathrm{C}$. It is assumed that the slight difference would not affect the investigations hereafter.

Table 1. Parameters for the selected BJT and MOSFET switches of three different ratings

\begin{tabular}{|c|c|c|c|c|c|}
\hline Switches & Type & Ratings $\left(V_{D S} / I_{D}\right.$ or $\left.V_{C E} / I_{C}\right)$ & $V_{t h}(\mathrm{~V})$ & $C_{g}(\mathrm{nF})$ & $C_{g} V_{t h}(\mathrm{nC})$ \\
\hline Si2392ads [32] & MOSFET & $100 \mathrm{~V} / 3.1 \mathrm{~A}$ & $1.2 \sim 3$, typically 2.3 & 0.1345 & 0.31 \\
\hline TIP31C $[33,34]$ & BJT & $100 \mathrm{~V} / 3 \mathrm{~A}$ & $<1.8$, typically 0.636 & 0.477 & 0.303 \\
\hline Si2308bds [35] & MOSFET & $60 \mathrm{~V} / 1.9 \mathrm{~A}$ & $1 \sim 3$, typically 1.9 & 0.133 & 0.253 \\
\hline PZT651T1 [36] & BJT & $60 \mathrm{~V} / 2 \mathrm{~A}$ & $<1$, typically 0.582 & 0.0745 & 0.043 \\
\hline SiUD402ed [37] & MOSFET & $20 \mathrm{~V} / 1 \mathrm{~A}$ & $0.4 \sim 0.9$, typically 0.62 & 0.01 & 0.006 \\
\hline BCP68T1 [38] & BJT & $20 \mathrm{~V} / 1 \mathrm{~A}$ & $<1$, typically 0.582 & 0.0745 & 0.043 \\
\hline
\end{tabular}

Beside the ratings, Table 1 also shows the parameters of the switches in different aspects: the parasitic capacitance $C_{g}\left(C_{g s}\right.$ for MOSFET and $C_{b e}$ for BJT), the turning-on threshold $V_{t h}\left(V_{g s-t h}\right.$ for MOSFET and $V_{B E-t h}$ for BJT), and the product of the two $C_{g} V_{t h}$. Since the parameters are not specifically given in the datasheet, the values used here are obtained from the Spice model provided by the manufacturer [32, 33] or the LTspice(C) library [34]. Moreover, the BJT's turning-on threshold $V_{B E-t h}$ adopts the base-emitter junction voltage $V_{j e}$ which is considered to be a reasonable estimation. It is close to the base-emitter voltage value with $I_{B}=1 \mathrm{~mA}$. Besides, the base-emitter capacitance $C_{b e}$ adopts the junction capacitance $C_{j e}$ as well. From table 1 , it is found that 
$V_{t h}$ and $C_{g}$ decreases with the maximum voltage and current ratings. Moreover, the MOSFET has larger $V_{t h}$ than the BJT does for higher ratings $(100 \mathrm{~V} / 3 \mathrm{~A}, 60 \mathrm{~V} / 2 \mathrm{~A})$ and the difference diminishes as the rating decreases $(20 \mathrm{~V} / 1 \mathrm{~A})$. In contrast, $C_{g}$ does not show a specific trend that the MOSFET or BJT has larger values than the other. It seems more likely dependent on the specific devices. In this paper, the comparison pair Si2392ads/TIP31C is used for following investigations due to the proper voltage rating, and more importantly, the close $C_{g} V_{t h}$ values as seen in Table 1.

Table 2. Brief comparison summary between BJT and MOSFET switches

\begin{tabular}{|c|c|c|c|}
\hline Definition & MOSFET & BJT & Preferred \\
\hline Gate parasitic capacitance $C_{g}$ & Depending on specific components & Depending on specific components & - \\
\hline Turning-on threshold $V_{t h}$ & Relatively Larger for high ratings & Relatively Small for high ratings & BJT \\
\hline Driving mechanism & Voltage-driven mechanism & Current-driven mechanism & MOSFET \\
\hline \multirow[t]{3}{*}{$C_{g}$ and $V_{t h}$ 's influence } & (1) Introduce more phase lag; & (1) Introduce less phase lag; & BJT \\
\hline & (2) Induce higher start voltage $V_{\text {start }}$ & (2) Induce smaller start voltage $V_{\text {start }}$ & \\
\hline & (3) Decrease $V_{o c}$ and $V_{a}$ & (3) Decrease $V_{o c}$ and $V_{a}$ & \\
\hline Driving mechanism's & (1) No requirement on $C_{p}$ & (1) Minimum requirement on $C_{p}$ to & MOSFET \\
\hline \multirow[t]{5}{*}{ influence } & (2) Slightly affect the energy transfer & ensure the saturation working region & \\
\hline & efficiency $\eta$ by the drain-source & (2) Significantly affect the efficiency $\eta$ & \\
\hline & resistance related to the gate-source & in the amplification region; & \\
\hline & voltage & (3) High $C_{p}$ decreases $V_{a}, V_{o c}$ and & \\
\hline & & increase the phase lag due to RC delay & \\
\hline
\end{tabular}

Among the parameters, $C_{g}$ and $V_{t h}$ affects the open-circuit voltage $V_{o c}$ and $\theta_{a}$. Easy to find from eq. (6), $V_{o c}$ decreases with $C_{g}$ so that $V_{a}$ gets smaller accordingly as indicated by eq. (2). Considering that $C_{g}$ is usually much smaller than $C_{0}$, $V_{o c}$ won't vary much for both switch types. In contrast, a more notable influence on the phase lag $\theta_{a}$ is exerted by $C_{g}$ and $V_{t h}$ together. Since the BJT and the MOSFET shows no obvious trend about $C_{g}$, the turning-on threshold $V_{t h}$ is mainly considered here. 
An additional effect related to $C_{g}$ and $V_{t h}$ is about the start voltage of the SP-OSECE circuit. In order to properly start the SP-OSECE, it requires the open-circuit voltage $V_{o c}$ to be capable of turning on the switches. It implies that

$$
V_{o c}-\Delta V_{1}-V_{B E}=\frac{V_{o c}-V_{D}}{\sqrt{1+\omega^{2} R_{p} C_{p}}}-V_{B E}>\Delta V_{2} \approx \frac{C_{g} V_{t h}}{C_{p}}+\frac{V_{t h} \theta_{3}}{\omega R_{g}}>\frac{C_{g} V_{t h}}{C_{p}}
$$

according to eq. (7) and eq. (8). When $\omega^{2} R_{p} C_{p}<<1$ is satisfied, it yields

$$
V_{o c}>C_{g} V_{t h} / C_{p}+V_{D}+V_{B E} \text { or } C_{p}>C_{g} V_{t h} /\left(V_{o c}-V_{D}-V_{B E}\right)
$$

It can be drawn that a minimum $V_{o c}$, called as the start voltage $V_{\text {start }}$, is required to start the SP-OSECE circuit for the given $C_{p}$ and the selected switches. In turn, a minimum requirement on $C_{p}$ also exists for a given $V_{o c}$ and selected switches. Due to relatively larger $V_{t h}$, a higher $V_{\text {start }}$ is needed for the MOSFET switch.

Beside $C_{g}$ and $V_{t h}$, the switch driven mechanism is another important factor of the SP-OSECE circuit performance. For MOSFET, the conduction of the switch is determined by the condition whether the gate-source voltage is larger than the threshold $V_{g s-t h}$. As indicated by the waveform $i_{g 1}$ in Fig. 3 (a) that no visible current is required at the gate, the MOSFET is a voltage-driven device and its performance is related to the drain-source resistance $R_{d s-o n}$, which is weakly dependent on the gate-source voltage as well. Here, for the Si2392ads in our case, $R_{d s-o n}$ differs from $0.1 \Omega \sim 0.4 \Omega$ with the gate-source voltage varying from $10 \mathrm{~V} \sim 4.5 \mathrm{~V}$ according to the datasheet [32]. High gate-source voltage leads to relatively low $R_{d s-o n}$, thus slightly improved circuit quality factor and energy transfer efficiency $\eta$. Distinguished from the MOSFET with the voltage-driven mechanism, the BJT switch is a current-driven device, which means that continual base current is necessary to keep on the conduction of the BJT switch even though the base-collector voltage is greater than the threshold voltage $V_{B E-t h}$. It is clearly seen in Fig. 3 (b) that a non-zero $i_{g 1}$ is injected into the base of the BJT. Depending on the base current, the BJT switch has three working regions: the amplification region with high DC current gain $\beta$ of the order of hundreds, the saturation region with low DC current gain $\beta$ of the order of dozens, and the cut-off region with ultra-low base current. When used as switches in the SP-OSECE circuit, the BJT is meant to work in the saturation region with small collector-emitter voltage $V_{C E}$ so that the power dissipation on the BJT switch is small for the purpose of high efficiency. It requires the base current $I_{B}$ is large enough to satisfy the following DC gain condition:

$$
\frac{I_{B}}{I_{C}}>\frac{1}{\beta_{c}}
$$


in which $I_{C}$ is the collector current and $\beta_{c}$ represents the assumed critical DC gain between the amplification region and the saturation region. To be noted, $\beta_{c}$ might vary with the BJT's working condition and not have a fixed value. Then, using the integration of $I_{B}$ and $I_{C}$ over the time of the whole voltage-inversion process, we can deduce the following equation

$$
C_{p}\left(V_{a}-V_{B E-t h}\right)>Q_{B}>\frac{1}{\beta_{c}} Q_{C} \approx \frac{1}{\beta_{c}} C_{0}\left(V_{a}+V_{\text {load }} / m\right)
$$

where $Q_{B}$ is the total charge into the base during the voltage inversion phase, $Q_{C}$ is the total charge into the collector. The last term at the right in eq. (12) represents the minimum charge on $C_{p i}$ to ensure that the BJT switch operates in the saturation region while the first term at the left represents the maximal charge available on $C_{p i}$ for the base current $I_{B}$. Especially, when both $V_{a}>>V_{B E-t h}$ and $V_{a}>>V_{\text {load }}$ are satisfied (which corresponds to most cases), we have

$$
C_{p}>\frac{1}{\beta_{c}} C_{0}
$$

It hints that a second minimum limit on $C_{p}$ beside eq. (8) exists for a given $C_{0}$ to ensure the working point of the selected BJT switch to be in the saturation region. In particular, this minimum limit is inversely proportional to the BJT critical DC gain $\beta_{c}$ and increases with $C_{0}$. On the one hand, the dissatisfaction of eq. (13) will lead to the shift of the BJT switch's working point into the amplification region with high collector-emitter voltage $V_{C E}$ so that the circuit energy transfer efficiency $\eta$ will significantly decrease. On the other hand, the increase of $C_{p}$ with respect to $C_{0}$ to satisfy eq. (13) decreases $V_{o c}$ with a factor of $2 C_{p}$ and increases $\theta_{1}+\theta_{2}$ simultaneously, resulting in smaller $V_{o c} \cos \theta_{a}$ in eq. (4). A trade-off on the determination of the optimal $C_{p}$ has to be made and the optimal $C_{p}$ is inclined to show strong correlation with $C_{0}$. It is especially unfavorable for large $C_{0}$ cases, in which a larger $C_{p}$ has to be used with the BJT switch while a smaller $C_{p}$ can be selected with the MOSFET with better performance in this point.

However, the current-driven mechanism of the BJT switch brings additional benefit on reducing the voltage retreatment $\Delta V_{3}$ as implied in Fig. 2, which is favorable to the performance according to eq. (3). In the MOSFET case, during the process of turning on the switch $S_{i}$, the voltage between $C_{p i}$ and $C_{g i}$ is almost balanced around $V_{r} \approx C_{p} V_{a} /\left(C_{p}+C_{g}\right)$ (denoted in Fig. 2 (a)) and the remaining charge on $C_{p i}$ to be neutralized can be calculated as $C_{p}^{2} V_{a} /\left(C_{p}+C_{g}\right)$ while the remaining charge of the other envelope capacitance is estimated as $C_{p} V_{p M}$. Then the voltage retreatment $\Delta V_{3}$ can be calculated according to the final voltage equilibrium between $C_{0}$ and the two capacitors $C_{p i}(i=1,2)$ after voltage inversion and we have 


$$
\Delta V_{3_{-} \text {Mos }} \approx \frac{V_{\text {load }}}{m}-\frac{C_{0} V_{\text {load }} / m-C_{p}^{2} V_{a} /\left(C_{p}+C_{g}\right)-C_{p} V_{p M}}{C_{0}+2 C_{p}+C_{g}}=\frac{C_{p}^{2} V_{a} /\left(C_{p}+C_{g}\right)+C_{p} V_{p M}+\left(2 C_{p}+C_{g}\right) V_{\text {load }} / m}{C_{0}+2 C_{p}+C_{g}}
$$

In the BJT case, after turning on the switches, the charge on $C_{p i}$ is totally injected to the base due to the current-driven mechanism and the remaining voltage $V_{r}$ and charge on $C_{p i}$ to be neutralized is zero as seen in Fig. 3 (b). Only the remaining charge on the other envelope detector capacitance $C_{p} V_{p M}$ is to be neutralized. Consequently, the voltage retreatment $\Delta V_{3}$ is calculated as

$$
\Delta V_{3 \_ \text {BJT }} \approx \frac{V_{\text {load }}}{m}-\frac{C_{0} V_{\text {load }} / m-C_{p} V_{p M}}{C_{0}+2 C_{p}+C_{g}}=\frac{C_{p} V_{p M}+\left(2 C_{p}+C_{g}\right) V_{\text {load }} / m}{C_{0}+2 C_{p}+C_{g}}
$$

which is much smaller than the value in the case of MOSFET. It can be also observed from the waveforms in Fig. 3 that the voltage on $C_{p i}$ after inversion is zero for the case of BJT switch and a large non-zero value of $V_{r}$ is found for the case of MOSFET.

From the above analysis, it is found that the switch type affects the circuit performance from various aspects and different features are found for both types respectively. The BJT's low $V_{B E-t h}$ promises both lower phase lag $\theta_{a}$ and smaller start voltage $V_{\text {start }}$ while the MOSFET can be used with smaller $C_{p}$ for large $C_{0}$ which is favorable to the circuit performance. However, it is interesting to find from Fig. 2 and Fig. 3 that, although the BJT switch possesses benefits of smaller phase lag $\theta_{\mathrm{a}}$ and voltage retreatment $\Delta V_{3}$, the circuit performance is still not as good as the MOSFET case. It implies that the current-driven mechanism of the BJT presents more critical limitations on the performance in this simulated case. In order to clarify the benefits and deficits of the two switch types and their influence on the circuit performance, further studies based on experiments and simulations have been performed and presented hereafter.

\subsection{Results}

To fulfil the comparative investigations about the two types of switches, a piezoelectric generator connected to the SP-OSECE circuit is prepared for experimental tests as shown in Fig. 4. It is composed of a steel cantilever beam $(20 \mathrm{~mm} \times 100 \mathrm{~mm} \times 0.6 \mathrm{~mm})$ and a piezoelectric ceramic patch $(20 \mathrm{~mm} \times 30 \mathrm{~mm} \times 0.4 \mathrm{~mm})$ attached to the beam top at the clamped end. A specially designed fixture is fabricated to fix the generator on a shaker (2075E-HT, The Modal Shop $\odot)$, which is driven by a signal source (DG1032, Rigol(C) through a power amplifier with a sinusoidal excitation of $67 \mathrm{~Hz}$ around the generator's resonant frequency. With the laser displacement sensor (HL-C203BE, SUNX@) adopted, the displacement amplitude of the beam tip is controlled to be constant as $u_{M}=1.25 \mathrm{~mm}$ by tuning the excitation level. Meanwhile, the voltage $V_{p}$ and $V_{\text {load }}$ are acquired by an oscilloscope. 


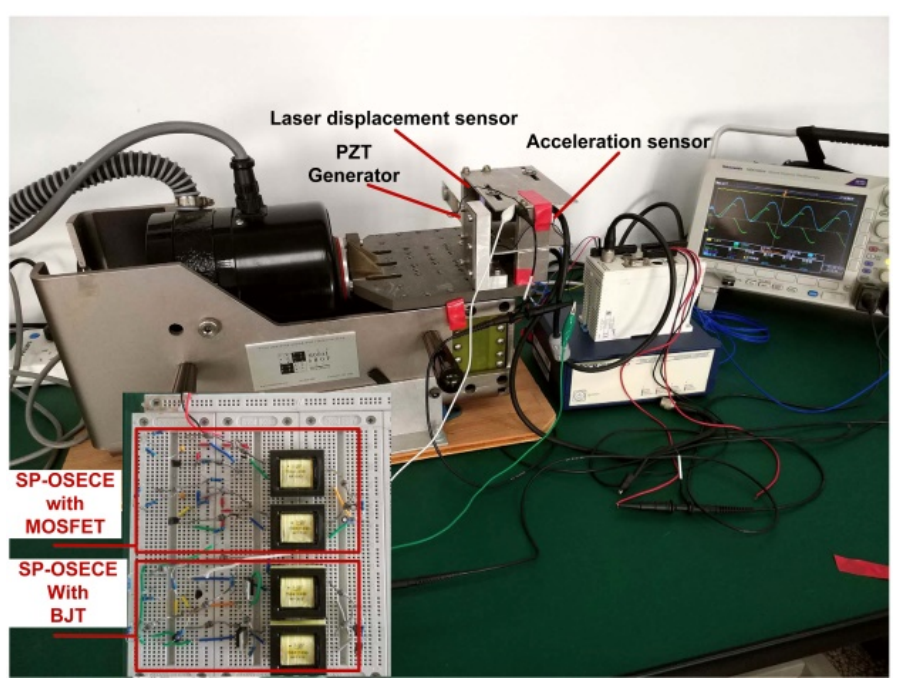

Fig. 4. Experimental set-up.

Table 3. Components and Parameters

\begin{tabular}{cccc}
\hline Definition & Value & Definition & Value \\
MOSFET $\left(S_{i}\right)$ & Si2392ads & BJT $\left(S_{i}\right)$ & TIP31C \\
Transformer $T_{1}$ & WE750811290 $(m=1)$ & Transistor $\left(T_{p i}\right)$ & $2 \mathrm{~N} 5401$ \\
Diodes $\left(D_{i}, D_{p i}\right)$ & $\mathrm{BYV} 28-100$ & $C_{0}$ & $13.75 \mathrm{nF}$ \\
$R_{0}$ & $5 \mathrm{M} \Omega$ & $R_{L}$ & $200 \mathrm{k} \Omega$ \\
$\alpha$ & $0.0003 \mathrm{~N} / \mathrm{V}$ & $R_{p i}$ & $200 \mathrm{k} \Omega$ \\
$R_{b i}$ & $3.3 \mathrm{k} \Omega$ & $R_{g i}$ & $1 \mathrm{M} \Omega$ \\
$f$ & $67 \mathrm{~Hz}$ & & $1.25 \mathrm{~mm}$ \\
\hline
\end{tabular}

Parameter identifications are firstly performed on the piezoelectric generator. With $C_{0}$ measured by a multi-meter, $\alpha$ and $R_{0}$ are determined through the original open-circuit voltage response without circuit and the phase difference between the displacement and the voltage. Table 3 lists the parameter values as well as the electronic components used in the SP-OSCECE circuit. With the equivalent current source determined using eq. (1), two simulation models using the MOSFET Si2392ads and the BJT TIP31C as switches are then established using the LT-SpiceC software. In the circuit, the resistor $R_{p i}$ in the envelope detector is selected to $200 \mathrm{k} \Omega$ for the purpose of avoiding misjudgment on the voltage extreme while the load resistor $R_{L}$ is fixed to be $200 \mathrm{k} \Omega$ for simplifying the investigations. However, it is thought that the load value wouldn't affect the comparison results with the same components since the load imposes identical effects on both cases according to eq. (1) and won't induce performance 
difference, especially considering the low load dependence of the SP-OSECE circuit [18]. Notably, the serial resistance $r$ is determined by fitting the simulations with experiments due to the difficulty of direct measurement.

As analyzed before, the SP-OSECE circuit's performance is closely related to the detector capacitance $C_{p}$. In view of that, multiple experiments and simulations with different $C_{p}$ have been performed for the circuit with the MOSFET and BJT switches respectively. Fig. 5 shows the results for these two cases. As seen in Fig. 5 (a), the obtained open-circuit voltage values $V_{o c}$ using two various switches are close to each other without obvious discrepancy and both decrease with the increasing $C_{p}$ values. It is logic according to eq. (6) since the parasitic capacitance $C_{g}$ is much smaller than $C_{0}$ with little effect on $V_{o c}$.
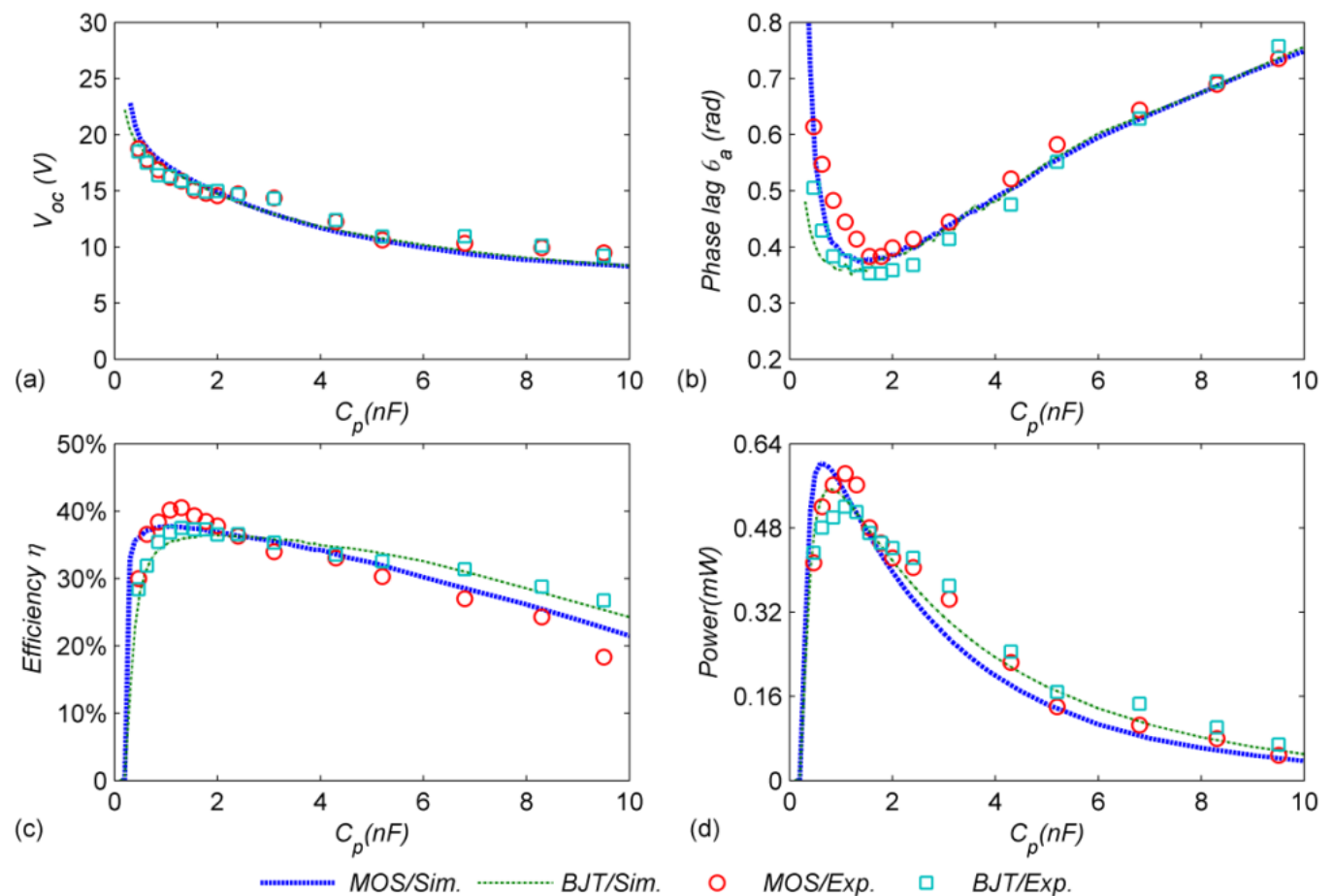

(d)

- MOS/Exp. BJT/Exp.

Fig. 5. Experimental and simulated results of the generator with SP-OSECE circuit using MOFFET Si2392ads and BJT TIP31C by varying $C_{p}$ : (a) open-circuit voltage $V_{o c}$; (b) phase lag $\theta_{a}$; (c) energy transfer efficiency $\eta$; (d) harvested power.

In contrast, obvious discrepancy is observed for the phase lag $\theta_{a}$ in the two cases as shown in Fig. 5 (b), especially for small $C_{p}$ values. The reason is that the MOSFET Si2392ads possesses larger $V_{t h}$ than the BJT TIP31C despite of similar product value of $C_{g} V_{t h}$. According to eq. (7) and eq. (8), a larger voltage drop $\Delta V_{2}$ is induced to turn on the switch Si2392ads, thus leading to the increase of $\theta_{3}$ and $\theta_{a}$. As $C_{p}$ increases, $\Delta V_{2}$ gets smaller according to eq. (8) and the proportion of $\theta_{3}$ in the total phase lag $\theta_{a}$ decreases while the phase lag $\theta_{a}$ is dominated by $\theta_{1}+\theta_{2}$ by virtue of the increased RC phase delay and the decreased $V_{o c}$ according to eq. (7). The close results of $\theta_{a}$ for large $C_{p}$ hint similar $\theta_{1}+\theta_{2}$ with the two different switches, in accordance with the 
aforementioned analysis. The same trend that the phase lag $\theta_{a}$ first decreases and then increases is found for both cases. It can be understood with eq. (7) and eq. (8) that, as $C_{p}$ increases, the dominant $\theta_{3}$ in $\theta_{a}$ for small $C_{p}$ cases decreases to the secondary position while the sum $\theta_{1}+\theta_{2}$ increases and begins to occupy the dominant position in $\theta_{a}$ for large $C_{p}$ values. Therefore, an optimal $C_{p}$ exists to obtain the smallest phase lag $\theta_{a}$ as shown in Fig. 5 (b).

As another important factor of the SP-OSECE circuit performance, the energy transfer efficiency $\eta$ is also plotted in Fig. 5 (c). Higher efficiency is obtained in the case of the MOSFET switch Si2392ads in comparison with the case of the BJT switch TIP31C for small $C_{p}$ values from $0.2 \mathrm{nF}$ to $2 \mathrm{nF}$ and the maximum efficiency in the MOSFET case is also higher than the one in the BJT case. It is because small $C_{p}$ values are not able to provide the required current to let the BJT switch work in the optimal saturation region. Consequently, larger power dissipation on the BJT switch decreases the energy transfer efficiency. As $C_{p}$ continually increases, the BJT works in the saturation region and better efficiency is found for BJT. The reason is that the BJT's benefit of reducing the voltage retreatment $\Delta V_{3}$ becomes obvious for large $C_{p}$ values with more charge to be neutralized.

Notably, the efficiency first increases then decreases with $C_{p}$ in both cases while the power performance shows the same trend as seen in Fig. 5 (d). It is partly due to the $C_{p}$ 's influence on the switch's conduction properties in the way of varying the working point of BJT or $R_{d s-o n}$ of MOSFET, and also partly due to the interaction between the load voltage and the efficiency itself. The power performance is related to the open-circuit voltage $V_{o c}$, the phase lag $\theta_{a}$ and the efficiency $\eta$ at the same time. As seen in Fig. 5 (a) and (b), too small $C_{p}$ introduces large phase lag (see Fig. 5 (b)) while too large $C_{p}$ decreases $V_{o c}$ and introduces more phase lag $\theta_{a}$ as well (see Fig. 5 (b) and (c)), leading to smaller $V_{a}$ for both small and large $C_{p}$ values according to eq. (3). The power performance would become worse correspondingly, implying smaller load voltage $V_{\text {load }}$ as well. When the voltage drop on the diodes, the serial resistance $r$ and the switches is comparable to $V_{\text {load }}$, the ratio of the energy consumed by these components during the voltage inversion and the energy transfer phases gets larger, leading to lower efficiency. In turn, the power performance is affected by the efficiency until the equilibrium achieves. Consequently, the efficiency $\eta$ shows the trend similar to the power performance in Fig. 5 (d).

By inspecting the power performance in Fig. 5 (d), it is found that the circuit using the MOSFET switch case obtains higher max performance, about $112 \%$ of the one with the BJT switch. Considering the larger phase lag introduced by the MOSFET 
Si2392ads and the similar $V_{o c}$ in both cases, it shows that the efficiency plays a critical role in the performance. More specifically,

the current-driven mechanism decreases the available maximum power due to the trade-off between $V_{o c} \cos \theta_{a}$ and $\eta$. As $C_{p}$ continually increases, the BJT in the saturation region brings superior power performance due to the less neutralized charge.
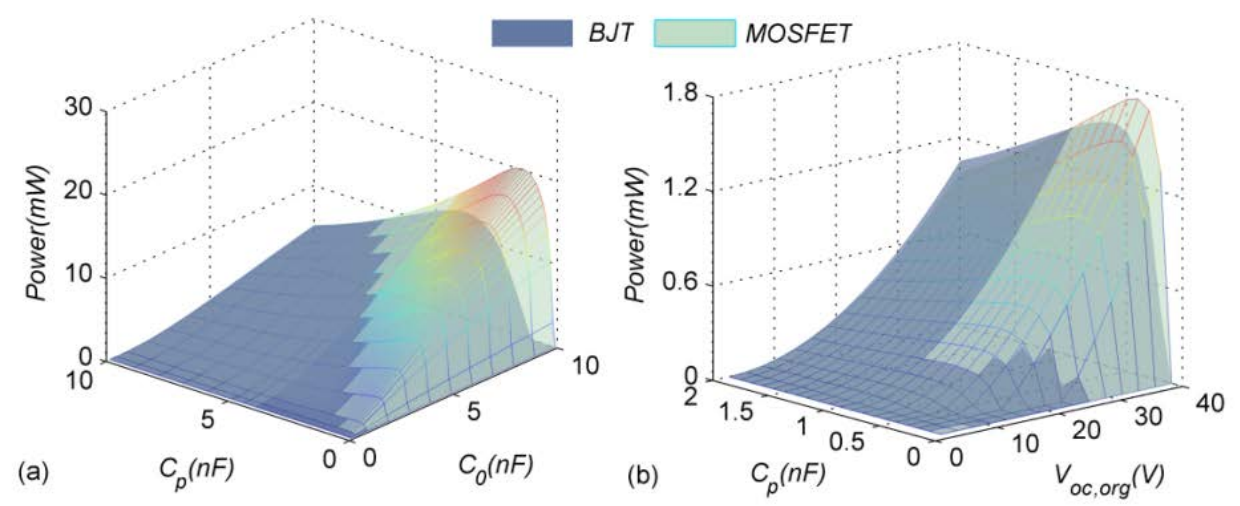

Fig. 6. Simulated power performance of the SP-OSECE circuit: (a) varying $C_{0}$ and $C_{p}$ with constant original open-circuit voltage $V_{o c, o r g}$; (b) Varying $V_{o c, o r g}$ and $C_{p}$ with $C_{0}$ constant.

(a)

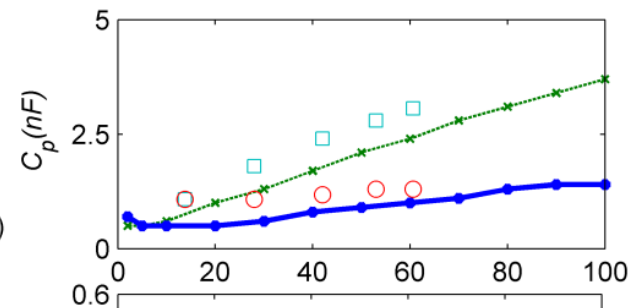

(c)

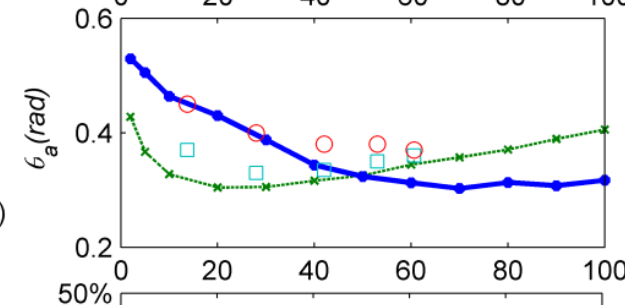

(e)

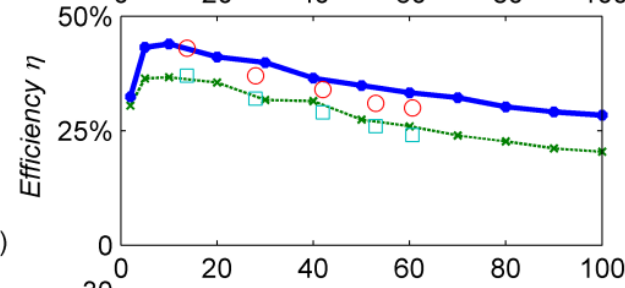

(g)

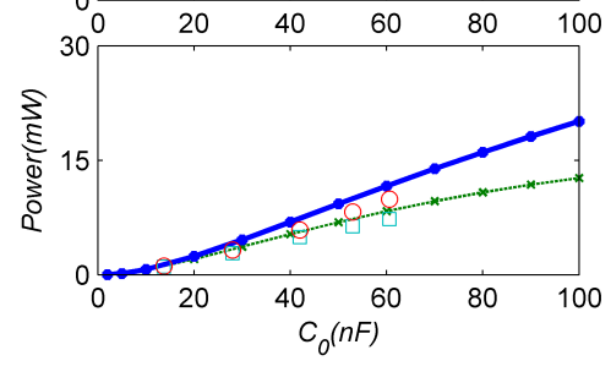

(b)

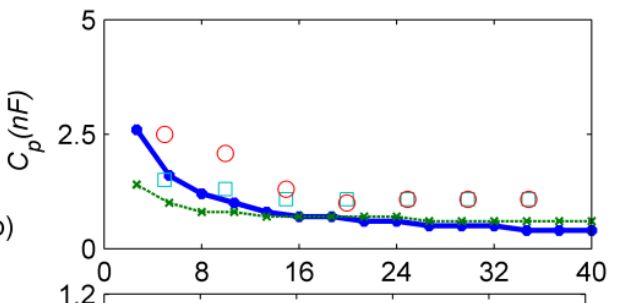

(d)

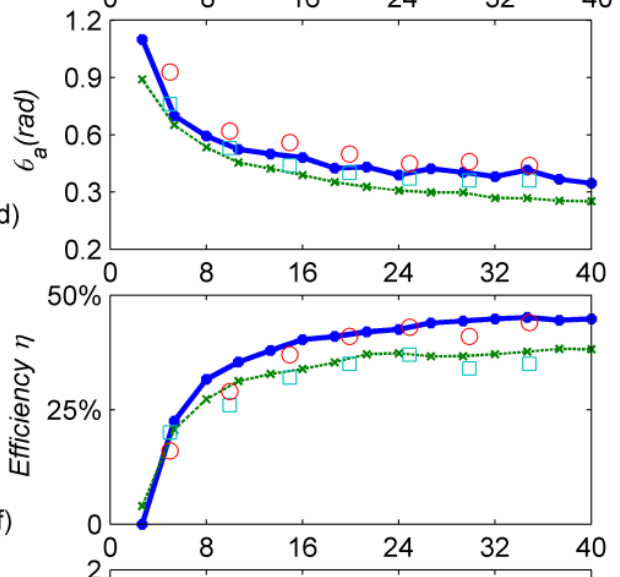

(h)

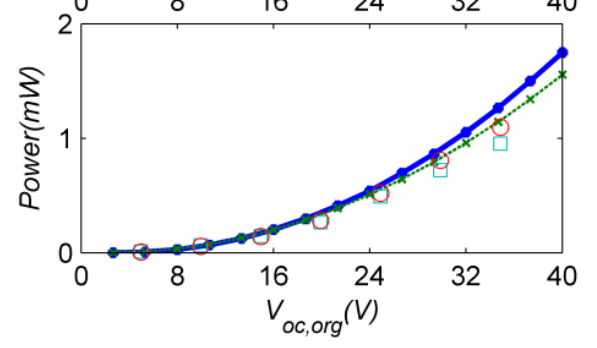

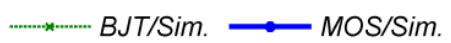

Fig. 7. The optimal power performance of the SP-OSECE circuit and the corresponding values of $C_{p}, \theta_{a}$ and $\eta$ : left column (a, c, e, g) varying $C_{0}$ with constant $V_{o c, o r g}$; right column (b, d, e, f) varying $V_{o c, o r g}$ with constant $C_{0}$.

Since a minimum $C_{p}$ is required to ensure the proper working region of the BJT switch for better efficiency and performance, it can be inferred that this minimum $C_{p}$ for BJT switches determined by eq. (13) increases with $C_{0}$. The effects of decreasing 
$V_{o c} \cos \theta_{a}$ will become more obvious making the obtained $V_{a}$ values smaller according to eq. (3). As a result, the available optimal performance using the BJT switch is inclined to be even worse in comparison with the case of using the MOSFET switch as $C_{0}$ increases. In addition, the performance comparison for different original open-circuit voltage $V_{o c, o r g}$ corresponding to different excitation levels is also meaningful to obtain a more complete and better understanding about the influence of the two different switch types. For these reasons, additional studies are performed for two typical cases: (a) generators with $C_{0}$ from small to large values with the same original open-circuit voltage $V_{o c, \text { org }}$ (b) the same generator $\left(C_{0}\right.$ constant) with $V_{o c, o r g}$ from small to large values. Consequently, a more complete comparison can be made for the two switch types. Considering that good agreements between experiment and simulation are validated in Fig. 5, the LT-Spice $\subset$ models are further used for the followed investigations.

Fig. 6 (a) shows the comparison results of the power performance when the generator's intrinsic capacitance $C_{0}$ varies from $2 \mathrm{nF}$ to $100 \mathrm{nF}$ with $V_{o c, o r g}$ constant as $37.5 \mathrm{~V}$ and $C_{p}$ varying from $0.1 \mathrm{nF}$ to $10 \mathrm{nF}$ for each $C_{0}$ case. It is shown he BJT switch brings almost the same optimal power as the MOSFET switch only in very small $C_{0}$ cases (no more than $5 \mathrm{nF}$ ). As $C_{0}$ increases, the MOSFET switch case outperforms the BJT switch case with much better power. However, for large $C_{p}$ values deviating from the optimal region of the power performance, the BJT brings slightly better performance than the MOSFET due to the benefit of less charge neutralization as analyzed in last section. Fig. 6 (b) presents the comparison results in the other case that the generator's original open-circuit voltage $V_{o c, o r g}$ varies from $2.7 \mathrm{~V}$ to $40 \mathrm{~V}$ with $C_{0}$ constant as $13.75 \mathrm{nF}$. It is seen that the BJT brings slightly better optimal performance for low $V_{o c, o r g}$ values while the MOSFET outperforms for large $V_{o c, o r g}$ values. The reason is that the phase lag introduced by BJT is smaller than the one introduced by MOSFET for small $V_{o c, o r g}$ values and it imposes more positive effects with the negative effects of the current-driven mechanism compensated.

In order to have a detailed view about the comparison, the optimal results in these two cases are plotted with the corresponding $C_{p}, \theta_{a}$ and $\eta$ depicted for the variable $C_{0}$ case (left column) and the variable $V_{o c, o r g}$ case (right column) in Fig. 7 . The following facts can be found that:

(1) As $C_{0}$ increases, the optimal $C_{p}$ increases correspondingly in the BJT case but stays almost stable in the MOSFET case as indicated in Fig. 7 (a). It is consistent with eq. (13) for the BJT case due to the current-driven mechanism. The very slight increase of the optimal $C_{p}$ in the voltage-driven MOSFET case is attributed to the lower phase lag (see Fig. 7 (c)) since increasing $C_{p}$ 
slightly will effectively decrease $\Delta V_{2}$ for relatively large $C_{0}$ values. Meanwhile, except the same decreasing trend for both cases at the beginning, the opposite trend is observed for the phase lag $\theta_{a}$ which increases with $C_{0}$ for the BJT case but decreases for the MOSFET case for relatively large $C_{0}$ case as shown in Fig. 7 (c). In the BJT case, the increasing optimal $C_{p}$ enlarges both the phase lag $\theta_{1}$ by introducing additional RC delay and the phase lag $\theta_{2}$ by lowering $V_{o c}$ so that $\theta_{a}$ increases. In the MOSFET case, with $V_{o c, o r g}$ constant and $C_{p}$ almost unvaried for the increasing $C_{0}$, the obtained open-circuit voltage $V_{o c}$ will increase according to eq. (4), leading to the decrease of $\theta_{2}$ and $\theta_{a}$ as well. Regarding the efficiency $\eta$ in Fig. 7 (e), the efficiency decreases with $C_{p}$ for both cases due to the circuit quality factor reduced with the increase of $C_{0}$. However, the decline speed of the efficiency in the BJT case is slightly faster than the speed in the MOSFET case. It is also attributed to the decrease of $V_{o c}$ induced by the larger optimal $C_{p}$ for the BJT switch. With the analysis above, it can be explained that the BJT switch brings similar performance for small $C_{0}$ but much worse performance for large $C_{0}$ in comparison with the MOSFET switch as indicated in Fig. 6 (a) and Fig. 7 (g), which is in accordance with the previous analysis in section 3.1. The optimal power with the MOSFET switch is $158.52 \%$ of the power with the BJT switch in the case of $C_{0}=100 \mathrm{nF}$.

(2) As seen in Fig. 7 (b), the optimal $C_{p}$ with the MOSFET is higher than the one with the BJT for small $V_{o c, o r g}$ cases since larger $\Delta V_{2}$ is required due to the higher $V_{t h}$ of Si2392ads. However, as $V_{o c, o r g}$ increases, the optimal $C_{p}$ decreases in both cases. Notably, the optimal $C_{p}$ with the MOSFET switch continues getting smaller and becomes lower than the one with the BJT switch which approaches to the minimum requirement of $C_{p}$ according to eq. (13). Meanwhile, the increasing $V_{o c, o r g}$ makes the phase lag induced by $\Delta V_{2}$ become smaller and negligible. Consequently, the phase lag $\theta_{\mathrm{a}}$ decreases as $V_{o c, o r g}$ increases and approaches a constant value and the MOSFET always brings larger phase lag as shown in Fig. 7 (d). In contrast, the efficiency increases in both cases to a stable value as indicated in Fig. 7 (f) since the dissipation on diodes becomes negligible for high $V_{\text {oc,org }}$ cases and the efficiency is mainly determined by the circuit quality factor related to the transformer and the switch. Due to the trade-off between the optimal $C_{p}$ and the efficiency induced by the current-driven mechanism, the BJT presents lower efficiency than the MOSFET for most cases and the difference is enlarged for large $V_{o c, o r g}$ cases. As a result, the optimal power in both cases is similar without obvious difference for small $V_{o c, o r g}$ while better results are found with the MOSFET switch for large $V_{o c, o r g}$ values. The power with the MOSFET switch is $112.41 \%$ of the power with the BJT switch for $V_{o c, o r g}=40 \mathrm{~V}$. 
For the purpose of validating the simulation results, experimental tests are performed on a generator with four piezoelectric patches. By varying the connected piezoelectric patch number and connecting an additional capacitance in parallel, the capacitance $C_{0}$ of the generator can be changed while the original open-circuit voltage is set to $37.5 \mathrm{~V}$ as simulation for the case of left column in Fig. 7. However, in order to avoid damage of the generator, the maximum capacitance tested in Fig. 7 is around $60 \mathrm{nF}$ since high displacement amplitude is required to obtain the open-circuit voltage of $37.5 \mathrm{~V}$ as $C_{0}$ increases. For the right column, a single piezoelectric patch is still used with the original open-circuit voltage changed by displacement amplitude. By checking the experimental results with the simulation, it is clear that the experimental results shows similar trend as the simulation and confirm the analysis well.

Experimental investigations are also performed about the SP-OSECE circuit's start voltage $V_{\text {start }}$, which is considered as an important figure about the circuit performance. It reflects when the circuit will start to work. Lower start voltage means the circuit can harvest energy from lower displacement amplitude $u_{M}$, corresponding to lower vibration level. Fig. 8 presents the experimental results of the SP-OSECE circuit with the two various types of switches. In both cases, $V_{\text {start }}$ decreases with $C_{p}$ as expected from eq. (10) since the voltage drop $\Delta V_{2}$ to turn on the switch become smaller. In particular, $V_{\text {start }}$ in the case of using the BJT switch is always smaller than the corresponding value in the case of using the MOSFET switch.

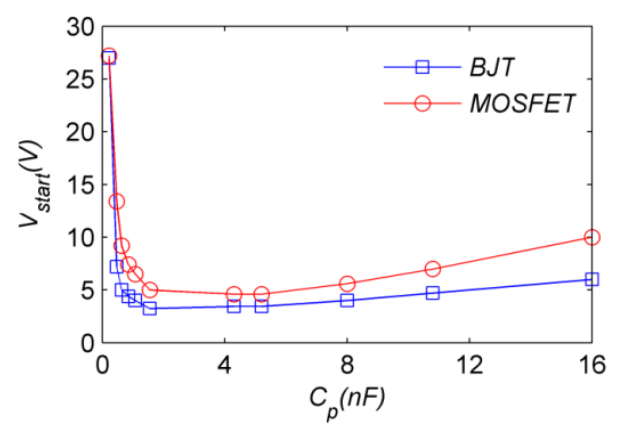

Fig. 8. Experimental start voltage of the SP-OSECE circuit with two different types of switches.

From the analysis above, it is shown that, due to relatively higher $V_{t h}$, the MOSFET Si2392 brings higher start voltage $V_{\text {start }}$ and larger phase lag than the BJT TIP31C. However, in comparison with the BJT TIP31C, similar performance is still obtained with the MOSFET Si2392 in smaller $C_{0}$ or $V_{o c, o r g}$ cases and much better performance is available for higher $C_{0}$ or $V_{o c, o r g}$ cases. It confirms that the current-driven mechanism of the BJT plays a critical role and restricts the optimal power performance to be worse than the one with the MOSFET of voltage-driven mechanism, especially for large $C_{0}$ or $V_{o c, o r g}$ cases. It can be further 
inferred that with identical $V_{t h}$ and $C_{g}$, the MOSFET would be always superior to the BJT in the SP-OSECE circuit with electronic breakers (optimal $C_{p}$ used) while $V_{\text {start }}$ would be the same.

\section{SP-OSECE circuit with external control units}

Beside the electronic breaker approach, the SP-OSECE circuit can also be realized with external control units, for instance, the velocity control approach [22] or using the integrated circuits [23, 24]. It is meaningful to investigate the switch type influence in these methods to provide a more complete comparison study. Generally, the external control unit can be represented by an equivalent pulse voltage source $V_{s}$ with a series resistor $R_{s}$ according to Thevenin's theorem as shown in Fig. 9. For simplifications, it is assumed that the low level output of $V_{s}$ is zero to turn off the switches and the high level output is $V_{H}$ to turn on the switches. Considering the relatively small values of $R_{s}$ (usually less than $100 \mathrm{k} \Omega$ ) and $C_{g}$ (in the order of $0.1 \mathrm{nF}$ ), the phase delay for turning on the switch is small and negligible.

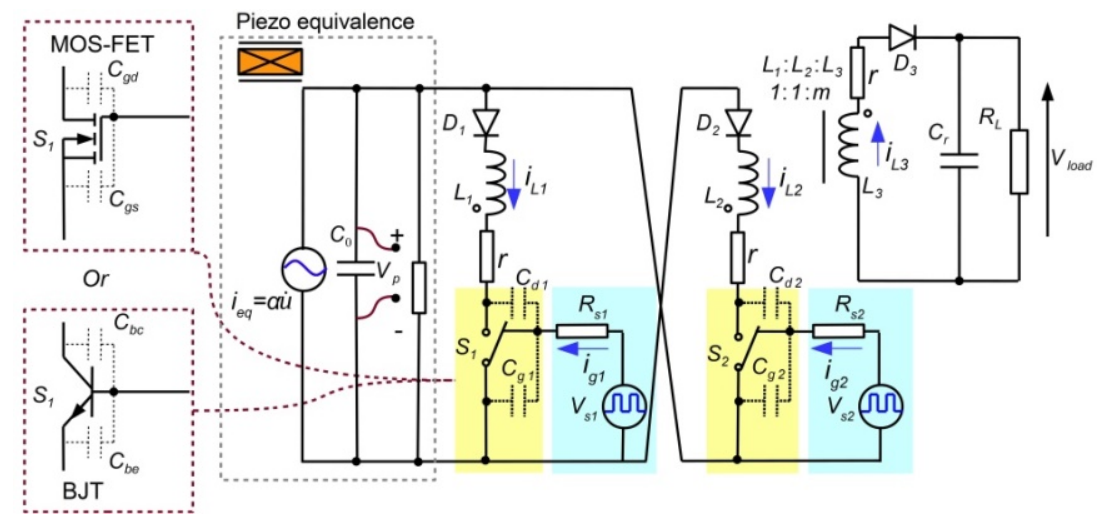

Fig. 9 Equivalent circuit for the SP-OSECE approach with external control circuit.
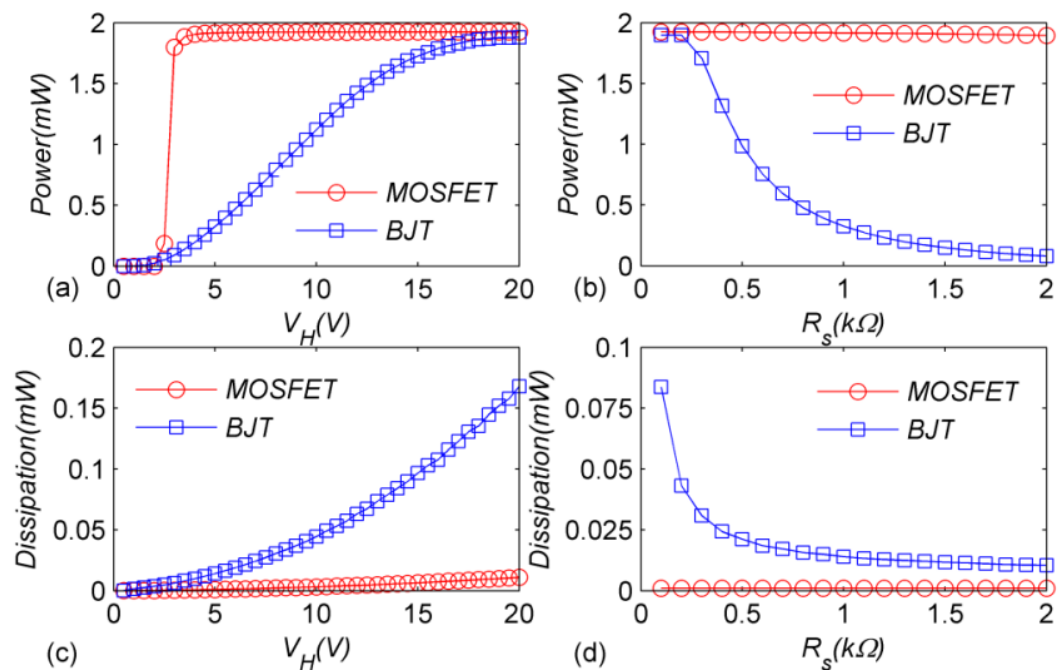

Fig. 10 Performance comparison between the SP-OSECE circuit with external control units and two different switches of the MOSFET Si2392ads and the BJT TIP31C: (a) power with $R_{s}=2 \mathrm{k} \Omega$ and variable $\mathrm{VH}$; (b) power with $V_{H}=5 \mathrm{~V}$ and variable $R_{s}$; (c) dissipation in the variable $V_{s}$ case; (d) dissipation in the variable $R_{s}$ case. 
Fig. 10 (a) shows the power performance for the case of $R_{s}=2 \mathrm{k} \Omega$ and variable $V_{H}$. This resistance value is selected according to the estimated results from the datasheet of TLV3701 [39] which was used in the velocity control method [22]. It is seen that the power output of the circuit using the BJT switch TIP31C starts for very small $V_{H}$ values and gradually increases to a stable value. It can be explained by the lower turning-on threshold $V_{t h}$ and the current-driven mechanism of the BJT since the small $V_{H}$ values is not enough to supply the current for the BJT in the saturation region. Therefore, the performance is limited unless $V_{H}$ is large enough as indicated in Fig. 10 (a). In contrast, the power output of the circuit with the MOSFET Si2392ads starts for a slightly larger $V_{H}$ value due to the higher $V_{t h}$ values but rapidly increases to the maximum value due to the voltage-driven mechanism. Fig. 10 (b) shows the performance for the other case of $V_{H}=5 \mathrm{~V}$ and variable $R_{s}$. Clearly, the performance of the circuit with MOSFET is almost unaffected while the power of the circuit with BJT decreases gradually with $R_{s}$ from a certain small value around $100 \Omega$. It implies that the current supplied by $V_{s}$ is not sufficient to provide the optimal working condition for the BJT TIP31C when $R_{s}$ increases. Obviously, the current-driven mechanism of the BJT makes the performance susceptible to external control circuits and special considerations are required for design.

To be noted, the power dissipation for the operation of the switches is another important factor to be considered. When the external control circuits are powered by additional independent sources, the power dissipation might be not so relevant. However, if the external control circuits are powered by the harvested power, the dissipation for operating the switches will play an important role in the final net power. For the MOSFET, the dissipation can be estimated as:

$$
P_{d_{-} m o s}=2 f C_{g} V_{H}^{2}
$$

which is used to charge $C_{g}$ to $V_{H}$ without continual current required. As for the BJT, it can be estimated as:

$$
P_{d_{-} B J T}=2 f \int_{0}^{T / 2} V_{s} i_{g} d t \approx 2 f V_{H}\left(C_{0} V_{p M} / \beta+C_{g} V_{t h}\right)
$$

Clearly, it is composed of two parts: (1) the consumption for turning on the BJT and (2) the consumption of keeping the BJT's conduction during the voltage inversion. Here, it is assumed the BJT is turned off exactly when the voltage inversion is finished. In order to make BJT work in the saturation region, we have $\beta>\beta_{c}$. Considering the normal case of $C_{g}<C_{0} / \beta_{c}$ and $V_{H}<V_{p M}, P_{d_{-} B J T}$ is inclined to be larger than $P_{d_{-} M O S}$. It is verified by the results in Fig. 10 (c) and (d) with the two different switches and other parts identical. It is found that the dissipation in the BJT case is much higher than the one in the MOSFET case. Moreover, the 
dissipation in the BJT case increases when $V_{H}$ increases or $R_{S}$ decreases. It is due to the increase of the gate current $i_{g}$. Meanwhile, dissipation in the MOSFET case increases slowly with $V_{H}$ but does not vary with $R_{S}$ as indicated in eq. (16).

It can be thus concluded that the MOSFET switch is a more favorable option for the SP-OSECE circuit with external control units in comparison with the BJT switch based on two reasons: (1) the facilitation of reaching stable maximum power and (2) less power dissipation for operation.

\section{Conclusion}

In this paper, comparative investigations have been performed about the influence of the switch type (BJT or MOSFET) on the performance of the SP-OSECE circuit. The analysis reveals that the performance difference comes from three aspects: the gate capacitance $C_{g}\left(C_{g s}\right.$ for MOSFET or $C_{b e}$ for BJT), the turning-on threshold voltage $V_{t h}\left(V_{g s-t h}\right.$ for MOSFET or $V_{B E-t h}$ for BJT $)$ and most importantly, the driven mechanism (the voltage-driven mechanism for MOSFET or the current-driven mechanism for BJT).

Preliminary survey on the available BJTs and MOSFETs for the power purpose shows that the MOSFET usually has higher turning-on threshold with the same voltage and current ratings while the gate capacitance does not have a specific trend. Followed investigations by experiments and simulations based on the case study of adopting the BJT TIP31C and the MOSFET Si2392ads show that

(1) For the SP-OSECE circuit with electronic breakers, the BJT switch has the advantage of lower start voltage $V_{\text {start }}$ and less phase lag for small $C_{0}$ cases (See Fig. 7) due to the relatively lower turning-on threshold $V_{t h}$. However, the current-driven mechanism of the BJT requires a minimum $C_{p}$ which increases with $C_{0}$ at the same time. In contrast, the voltage-driven mechanism of the MOSFET only requires $C_{p}$ to charge the gate capacitance $C_{g}$ and turn on the switch with small phase lag in consideration of the trade-off for $V_{o c}$. The optimal $C_{p}$ in the MOSFET case shows weak dependence on $C_{0}$ and decreases as $V_{o c, o r g}$ increases. Consequently, small optimal $C_{p}$ values are obtained in the MOSFET case. It makes the circuit with the MOSFET switch shows much better results on the available optimal power than the one with the BJT switch for large $C_{0}$ cases. Moreover, the optimal power performance using the MOSFET switch is close to the one with the BJT switch even in the small $C_{0}$ cases.

(2) For the SP-OSECE circuit with external control units, the BJT switch always show worse performance than the 
MOSFET except smaller start voltage. The current-driven mechanism requires the external unit to provide higher driving current to let the BJT work in the saturation region. Otherwise, the performance degenerates rapidly. Meanwhile, the MOSFET shows stable performance which are less affected by external control circuits.

With the same voltage and current ratings, it can be therefore concluded that the MOSFET switch is superior to the BJT one for most aspects except the start voltage. Finally, it is reminded that the investigations are performed on the SP-OSECE circuit using the BJT or MOSFET switches, but can be extended to other self-powered synchronous circuits and other switch types of current or voltage driven mechanism with the same operations.

\section{Acknowledgements}

The authors would like to thank the support from National Natural Science Foundation of China (NSFC) under the grant No.51505395, Science \& Technology department of Sichuan (2016HH0028), China Postdoctoral Science Foundation (2017T100709, 2016M600747) and the Fundamental Research Funds for the Central Universities (2682017CX26, 2682017CY05).

\section{References}

[1] Y. Zhang, F. Zhang, Y. Shakhsheer, J. D. Silver, A. Klinefelter, M. Nagaraju, S. Member, J. Boley, J. Pandey, A. Shrivastava, E. J. Carlson, A. Wood, B. H. Calhoun, S. Member, and B. P. Otis, "A batteryless 19 W MICS/ISM-band energy harvesting body sensor node soc for EXG applications,” IEEE J. Solid-State Circuits, vol. 48, no. 1, pp. 199-213, 2013.

[2] W. Li, S. He, and S. Yu, "Improving power density of a cantilever piezoelectric power harvester through a curved L-shaped proof mass," IEEE Trans. Ind. Electron., vol. 57, no. 3, pp. 868-876, Mar. 2010.

[3] W. Liu, C. Liu, B. Ren, Q. Zhu, G. Hu, and W. Yang, "Bandwidth increasing mechanism by introducing a curve fixture to the cantilever generator," Appl. Phys. Lett., vol. 109, p. 43905, 2016.

[4] L. Tang, Y. Yang, and C. Soh, “Toward broadband vibration-based energy harvesting,” J. Intell. Mater. Syst. Struct., vol. 21, no. 18, p. 1867, 2010.

[5] D. Zhu, M. J. Tudor, and S. P. Beeby, "Strategies for increasing the operating frequency range of vibration energy harvesters: a 
review," Meas. Sci. Technol., vol. 21, no. 2, p. 22001, Feb. 2010.

[6] G. K. Ottman, H. F .Hofmann, A. C. Bhatt, and G. A. Lesieutre, “Adaptive piezoelectric energy harvesting circuit for wireless remote power supply,” IEEE Trans. Power Electron., vol. 17, no. 5, pp. 669-676, Sep. 2002.

[7] J. R. Liang and W. H. Liao, "Piezoelectric energy harvesting and dissipation on structural damping," J. Intell. Mater. Syst. Struct., vol. 20, no. 5, pp. 515-527, Mar. 2009.

[8] E. Lefeuvre, A. Badel, C. Richard, L. Petit, and D. Guyomar, “A comparison between several vibration-powered piezoelectric generators for standalone systems,” Sensors Actuators A Phys., vol. 126, no. 2, pp. 405-416, Feb. 2006.

[9] D. Guyomar, A. Badel, E. Lefeuvre, and C. Richard, “Toward Energy Harvesting Using Active Materials and Conversion Improvement by Nonlinear Processing,” IEEE Trans. Ultrason. Ferroelectr. Freq. Control, vol. 52, no. 4, pp. 584-595, 2005.

[10] I. C. Lien, Y. C. Shu, W. J. Wu, S. M. Shiu, and H. C. Lin, "Revisit of series-SSHI with comparisons to other interfacing circuits in piezoelectric energy harvesting,” Smart Mater. Struct., vol. 19, no. 12, p. 125009, Dec. 2010.

[11] A. Badel, A. Benayad, E. Lefeuvre, L. Lebrun, C. Richard, and D. Guyomar, "Single crystals and nonlinear process for outstanding vibration-powered electrical generators," IEEE Trans. Ultrason. Ferroelectr. Freq. Control, vol. 53, no. 4, pp. $673-683,2006$.

[12] E. Lefeuvre, ] A. Badel, C. Richard, and D. Guyomar, "Piezoelectric Energy Harvesting Device Optimization by Synchronous Electric Charge Extraction,” J. Intell. Mater. Syst. Struct., vol. 16, no. 10, pp. 865-876, Oct. 2005.

[13] M. Lallart, L. Garbuio, L. Petit, C. Richard, and D. Guyomar, "Double synchronized switch harvesting (DSSH): A new energy harvesting scheme for efficient energy extraction," IEEE Trans. Ultrason. Ferroelectr. Freq. Control, vol. 55, no. 10, pp. 2119-2130, 2008.

[14] L. Garbuio, M. Lallart, D. Guyomar, C. Richard, and D. Audigier, "Mechanical Energy Harvester With Ultralow Threshold Rectification Based on SSHI Nonlinear Technique,” IEEE Trans. Ind. Electron., vol. 56, no. 4, pp. 1048-1056, Apr. 2009.

[15] H. Shen, J. Qiu, H. Ji, K. Zhu, and M. Balsi, “Enhanced synchronized switch harvesting: a new energy harvesting scheme for efficient energy extraction,” Smart Mater. Struct., vol. 19, no. 11, p. 115017, Nov. 2010.

[16] Y. Wu, A. Badel, F. Formosa, W. Liu, and A. E. Agbossou, "Piezoelectric vibration energy harvesting by optimized 
synchronous electric charge extraction,” J. Intell. Mater. Syst. Struct., vol. 24, no. 12, pp. 1445-1458, Dec. 2012.

[17] E. Lefeuvre, A. Badel, A. Brenes, S. Seok, M. Woytasik, and C.-S. Yoo, "Analysis of piezoelectric energy harvesting system with tunable SECE interface,” Smart Mater. Struct., vol. 26, no. 3, 2017.

[18] Y. P. Wu, A. Badel, F. Formosa, W. Q. Liu, and A. Agbossou, "Nonlinear vibration energy harvesting device integrating mechanical stoppers used as synchronous mechanical switches," J. Intell. Mater. Syst. Struct., vol. 25, no. 14, pp. 1658-1663, Apr. 2014.

[19] W. Liu, F. Formosa, A. Badel, Y. Wu, and A. Agbossou, "Self-powered nonlinear harvesting circuit with a mechanical switch structure for a bistable generator with stoppers," Sensors Actuators A Phys., vol. 216, pp. 106-115, Sep. 2014.

[20] H. Liu, J. Liang, and C. Ge, “A mechatronic power boosting design for piezoelectric generators," Appl. Phys. Lett., vol. 107, no. 14, p. $141902,2015$.

[21] Y. S. Shih, D. Vasic, and W. J. Wu, “A non-contact mechanical solution for implementing synchronized switching techniques for energy harvesting using reed switches," Smart Mater. Struct., vol. 25, no. 12, p. 125013, 2016.

[22] Y. Y. Chen, D. Vasic, F. Costa, W. J. Wu, and C. K. Lee, "A self-powered switching circuit for piezoelectric energy harvesting with velocity control,” Eur. Phys. J. Appl. Phys., vol. 57, no. 3, p. 30903, Feb. 2012.

[23] S. Lu and F. Boussaid, "A Highly Efficient P-SSHI Rectifier for Piezoelectric Energy Harvesting," IEEE Trans. Power Electron., vol. 30, no. 10, pp. 5364-5369, 2015.

[24] S. Boisseau, P. Gasnier, M. Gallardo, and G. Despesse, "Self-starting power management circuits for piezoelectric and electret-based electrostatic mechanical energy harvesters,” J. Phys. Conf. Ser., vol. 476, p. 12080, 2013.

[25] N. Kong and D. S. Ha, "Low-power design of a self-powered piezoelectric energy harvesting system with maximum power point tracking," IEEE Trans. Power Electron., vol. 27, no. 5, pp. 2298-2308, 2012.

[26] M. Lallart and D. Guyomar, “An optimized self-powered switching circuit for non-linear energy harvesting with low voltage output,"Smart Mater. Struct., vol. 17, no. 3, p. 35030, 2008.

[27] J. Liang and W. Liao, "Improved design and analysis of self-powered synchronized switch interface circuit for piezoelectric energy harvesting systems," IEEE Trans. Ind. Electron., vol. 59, no. 4, pp. 1950-1960, 2012. 
[28] Y. Wu, A. Badel, F. Formosa, W. Liu, and A. Agbossou, "Self-powered optimized synchronous electric charge extraction circuit for piezoelectric energy harvesting,” J. Intell. Mater. Syst. Struct., vol. 25, no. 17, pp. 2165-2176, 2014.

[29] W. Q. Liu, A Badel, F. Formosa, Q. Zhu, C. Zhao and G. Hu, “A Comprehensive Analysis and Modeling of the Self-powered Synchronous Switching Harvesting Circuit with Electronic Breakers,” IEEE Trans. Ind. Electron., online first, 2017. Doi: 10.1109/TIE.2017.2762640.

[30] W. Q. Liu, A. Badel, F. Formosa, Y. P. Wu, and A. Agbossou, "Wideband energy harvesting using a combination of an optimized synchronous electric charge extraction circuit and a bistable harvester," Smart Mater. Struct., vol. 22, no. 12, pp. $125038,2013$.

[31] W. Liu, A. Badel, F. Formosa, C. Liu, and G. Hu, “An improved switching control law for the optimized synchronous electric charge extraction circuit,” J. Phys. Conf. Ser., vol. 660, p. 12097, 2015.

[32] http://www.vishay.com/mosfets/list/product-62960.

[33] http://www.st.com/content/st_com/en/products/power-transistors/power-bipolar/lt-500v-transistors/tip31c.html

[34] http://ltwiki.org/index.php5?title=Standard.bjt.

[35] http://www.vishay.com/mosfets/list/product-69958/

[36] http://www.onsemi.cn/PowerSolutions/product.do?id=PZT651T1

[37] http://www.vishay.com/product?docid=62968

[38] http://www.onsemi.cn/PowerSolutions/product.do?id=BCP68T1

[39] http://www.ti.com.cn/product/cn/TLV3701 